\title{
ЗАВИСИМОСТЬ РЕЗУЛЬТАТОВ РАДИКАЛЬНОГО ЛЕЧЕНИЯ БОЛЕЗНИ ИЦЕНКО-КУШИНГА ОТ МР-КАРТИНЫ ГИПОФИЗА У ДЕТЕЙ
}

\author{
Э.А. Янар, Н.В. Маказан, М.А. Карева, В.А. Петеркова \\ ФГБУ «Начиональный медицинский исследовательский чентр эндокринологии» Минздрава \\ России, Москва, Россия
}

ВВЕДЕНИЕ. Болезнь Иценко-Кушинга (БИК) - крайне редкое заболевание, причиной которого является хроническая гиперпродукция кортизола вследствие стимуляции АКТГ, секретируемого аденомой гипофиза (кортикотропиномой). Стандартом терапии является хирургический метод лечения (ХЛ), который в 70-90\% случаев приводит к ремиссии заболевания. В случае неэффективности ХЛ проводится лучевая терапия. Предоперационные параметры прогнозирования возможных исходов ХЛ на сегодняшний день не установлены, что делает востребованными исследования потенциальных предикторов эффективности.

ЦЕЛЬ - оценка эффективности радикальных методов лечения в зависимости от визуализации и размера кортикотропином в педиатрической практике.

МАТЕРИАЛЫ И МЕТОДЫ. Ретроспективное исследование исходов болезни Иценко-Кушинга у 91 ребенка (46 девочек [51\%], 45 мальчиков [49\%]), наблюдавшихся в период с 1992г по 2020 г в ФГБУ «НМИЦ эндокринологии» Минздрава России. Пациенты были разделены на 3 группы в зависимости от МР-картины: группа 1 - пациенты с невизуализируемой аденомой [n=37], группа 2 - пациенты с микроаденомой гипофиза (<10 мм) [n=41], группа 3 - пациенты с макроаденомой (>10 мм) [n=13]. В 68\% случаев [62/91] было проведено трансназальное удаление аденомы гипофиза, в 32\% - лучевая терапия [29/91] (28 пациентов получили протонотерапию, 1 пациент - стереотаксическую радиохирургию на установке гамма-нож). Эффективность хирургического метода лечения (ХЛ) подтверждалась в раннем послеоперационном периоде при достижении гипокортицизма (уровни кортизола менее 50 нмоль/л). У пациентов, получивших лучевую терапию (Лт) в качестве 1 этапа терапии, эффективность оценивалась не раньше, чем через 6 мес. по нормализации или снижению уровня свободного кортизола в суточной моче ( $<400$ нмоль/сут). Рецидив заболевания подтверждался при развитии клинико-лабораторной картины гиперкортицизма после подтвержденной эффективности лечения.

РЕЗУЛЬТАТЫ. В 1 группе 49\% пациентов [18/37] получили ХЛ, и из них у 61\% пациентов терапия была эффективна [11/18]; 51\% пациентов [19/37] получили ЛТ, ремиссия была достигнута у 84\% пациентов [16/19]. В 2 группе пациентов 80\% получили ХЛ [33/41], и у 79\% [26/33] достигнута ремиссия; 19\% [8/41] получили ЛТ, ремиссия достигнута у 75\% [6/8] пациентов. В 3 группе пациентов 85\% [11/13] получили ХЛ, и у 73\% [8/11] терапия была эффективна; 15\% [2/13] получили ЛТ, ремиссия достигнута у 100\% [2/2] пациентов. Среди пациентов с ремиссией заболевания после первого этапа лечения [69/91] рецидив произошел у одного из 27 пациентов из 1 группы [3,7\%, у 2 пациентов из 2 группы [25\%] и у 2 пациентов из 3 группы [20\%]. Различия частоты случаев рецидива в группах статистически незначимы (

Выводы. Статистически значимой связи МРТ-характеристик кортикотропином с исходами эффективностью лечения и вероятностью развития рецидива выявлено не было. Вопрос дальнейшего поиска и анализа предикторов ремиссии и рецидива кортикотропином остается актуальным. 